\title{
Locating and Quantifying Carbon Steel Corrosion Rates Linked to Fungal B20 Biodiesel Degradation
}

James G. Floyd ${ }^{1}$, Blake W. Stamps ${ }^{2,3}$, Wendy J. Goodson ${ }^{4}$, Bradley S. Stevenson ${ }^{1}$

1 - University of Oklahoma, Department of Microbiology and Plant Biology, Norman OK 2 - 711th Human Performance Wing, Airman Systems Directorate, Air Force Research Laboratory, Wright-Patterson AFB, OH, United States

3 - UES Inc., Integrative Health and Performance Sciences Division, Dayton, $\mathrm{OH}$, United States

4 - Soft Matter Material Branch, Materials and Manufacturing Directorate, Air Force Research Laboratory, Wright-Patterson AFB, OH

Key Words: Biocorrosion, Fungi, B20 Biodiesel, Biodegradation, Carbon Steel, Pitting

16 Corrosion, Surface Analysis. 


\section{ABSTRACT}

Fungi that degrade B20 biodiesel in storage tanks have also been linked to

45 microbiologically influenced corrosion (MIC). A member of the filamentous fungal genus

46 Byssochlamys, and a yeast from the genus Wickerhamomyces were isolated from

47 heavily contaminated B20 storage tanks from multiple Air Force bases. Although these

48 taxa were linked to microbiologically influenced corrosion in situ, precise measurement

49 of their corrosion rates and pitting severity on carbon steel was not available. In the

50 experiments described here, we directly link fungal growth on B20 biodiesel to higher

51 corrosion rates and pitting corrosion of carbon steel under controlled conditions. When

52 these fungi were growing solely on B20 biodiesel for carbon and energy, consumption

53 of FAME and n-alkanes was observed. The corrosion rates for both fungi were highest

54 at the interface between the B20 biodiesel and the aqueous medium, where they

55 acidified the medium and produced deeper pits than abiotic controls. Byssochlamys

56 produced the most corrosion of carbon steel and produced the greatest pitting damage.

57 This study characterizes and quantifies the corrosion of carbon steel by fungi that are

58 common in fouled B20 biodiesel through their metabolism of the fuel, providing valuable

59 insight for assessing MIC associated with storage and dispensing B20 biodiesel.

\section{IMPORTANCE}

61 Biodiesel is widely used across the United States and worldwide, blended with

62 ultralow sulfur diesel in various concentrations. In this study we were able to

63 demonstrate that the filamentous fungi Byssochlamys AF004 and the yeast

64 Wickerhamomyces SE3 were able to degrade fatty acid methyl esters and alkanes in

65 biodiesel causing increases in acidity. Both fungi also accelerated the corrosion of

66 carbon steel, especially at the interface of the fuel and water, where their biofilms were 
67 located. This research provides controlled, quantified measurements and the

68 localization of microbiologically influenced corrosion caused by common fungal

69 contaminants in biodiesel fuels.

\section{INTRODUCTION}

71 Biodiesel production within the United States (U.S.) greatly expanded in

72 response to high petroleum prices and an increased need for energy security after the

73 September 11, 2001 terrorist attacks $(1,2)$. Over two decades afterwards, world

74 biodiesel consumption has continued to increase, reaching 9.3 billion gallons in 56

75 countries in 2016 (3). Consumption in the U.S. increased from 322 million gallons

76 annually in 2009 to 1.8 billion gallons in 2019 (4). The U.S. increase in biodiesel

77 consumption was largely driven by an increased interest in energy security but there are

78 other advantages to its' use. When biodiesel is blended with or used as an additive to

79 ultra-low sulfur diesel (ULSD), fuel lubricity is restored and emissions of carbon and

80 particulates are partially offset $(5,6)$. The increased energy independence and fuel

81 performance resulted in widespread adoption of biodiesel blends worldwide. The largest

82 energy consumer in the U.S., the United States Department of Defense (DoD), sought

83 to diversify its' energy supplies to reduce fuel security risk through the use of renewable

84 'drop-in' fuels to replace unblended fuels such as ULSD (7). The United States Air

85 Force (USAF), a service within the DoD, rapidly implemented the use of a drop-in fuel in

86 the form of a $20 \%$ first-generation biodiesel blend (B20) in non-tactical ground vehicles

87 and equipment. B20 is composed of a $20: 80$ volume/volume blend of fatty acid methyl

88 esters (i.e. FAME biodiesel) and ULSD, which is compatible with existing engines and

89 storage infrastructure (8). The FAME used as biodiesel is a renewable resource 
90 produced through an esterification reaction with animal and plant triglycerides and

91 methanol (9). However, the advantages that adoption of B20 represent are tempered

92 by potential limitations.

93 Biodiesel is more hygroscopic and oxidatively unstable compared to petroleum-

94 based diesel, making fuels containing biodiesel more susceptible to microbiological

95 degradation (10). This is especially true during long term storage as microorganisms

96 can readily degrade the FAME in B20 (11). The half-life of the FAME in B20, as well as

$97 \mathrm{C} 7$ to $\mathrm{C} 20$ alkanes were 2.1 to 2.8 days in aqueous microcosm experiments, suggesting

98 that both the FAME and alkanes in biodiesel blends is a viable oxidizable substrate for

99 microbial growth (12). The biodegradation of FAME is carried out through the $\beta$ -

100 oxidation pathway, which involves the sequential removal of two-carbon components

101 and production of acetic acid. Acetic acid can then be converted to acetyl-CoA and used

102 in the Krebs cycle or exported from the microbial cell (13-15). Methanol produced from

103 the de-esterification of FAME is readily metabolized by acid-tolerant fungi and bacteria

104 (16). The aliphatic alkanes in B20 are also readily degraded by both fungi and bacteria

105 suggesting that the oxidation of B20 under aerobic conditions is highly favorable (17,

106 18). Under aerobic conditions alkanes are oxidized by alkane monooxygenases

107 producing a fatty acid that is shuttled into the $\beta$-oxidation pathway, producing additional

108 acetic acid (19). Free fatty acids and acidic byproducts produced from the metabolism

109 of many of the components of B20 fuel can acidify both the fuel and any water present

110 within the storage tank, inducing or accelerating corrosion, leading to damaged vehicles

111 and storage infrastructure. 
Corrosion directly or indirectly caused by microorganisms is known as

113 microbiologically influenced corrosion, or MIC. Annually, corrosion costs an estimated

1142.5 trillion dollars worldwide, up to $20 \%$ of which is attributed to MIC (20). MIC is

115 initiated at the interface of microorganisms, often in the form of a biofilm, and material

116 surfaces. Biofilms affect the physical and chemical environment of metallic surfaces,

117 impacting the kinetics of cathodic or anodic reactions $(21,22)$. Microorganisms in

118 biofilms can also secrete enzymes that attack metals, increase local acidity, create

119 differential aeration, and form galvanic cells which accelerate corrosion under otherwise

120 aerobic conditions (23).

$121 \quad$ Fungi can increase corrosion rates of mild steel when grown on ULSD as a sole

122 carbon and energy source (24). Fungi grow more rapidly and produce more biomass on

123 the FAME in biodiesel blends than ULSD, which could lead to a greater corrosion risk to

124 infrastructure storing or dispensing fuels containing biodiesel (25). Filamentous fungi

125 such as Penicillium and Aspergillus increase the rate of steel corrosion when degrading

126 diesel fuel (26). It was speculated that the increased steel corrosion rates from these

127 fungi degrading diesel were likely attributed to increased oxygen concentrations in the

128 medium caused by degrading benzene rings and aliphatic hydrocarbons to (-O- $\left.\mathrm{CH}_{2}-\right)$.

129 The authors also acknowledged that these organisms were likely producing organic

130 acids that could also play a role in the increased corrosion risk. Additionally, studies

131 have shown that fungi contaminating biodiesel storage tanks in Brazil were able to

132 degrade fuels containing 5\%,10\%, 20\% and $100 \%$ biodiesel (25). Although they did not

133 measure corrosion during their investigation, the authors did note that as fungi

134 degraded these fuels, there was an increase in acidity that could impact corrosion risks. 
135 There is a critical need to provide quantified rates and measurements of how fungi can

136 degrade fuels, such as B20, and subsequently contribute to MIC in critical fuel storage

137 infrastructure.

138 When the USAF started using B20 to meet mandated energy requirements,

139 problems quickly arose (27). Numerous USAF bases reported particulates in the fuel

140 from B20 storage tanks and fouled filters on dispensers (28). Subsequent in-situ

141 analysis of corrosion confirmed that tanks with obvious fungal contamination had

142 significant pitting corrosion relative to controls. These storage tanks being monitored

143 were still in operation making it difficult to control for other factors that might have

144 contributed to the perceived increase in corrosion risk. Herein we describe the isolation

145 of organisms responsible for fouling contaminated B20 storage tanks and directly link

146 them to fuel degradation and increased corrosion risks. To address our lack of a direct

147 causative link between the previously identified fungal taxa within USAF B20 tanks and

148 their ability to both increase corrosion rates of carbon steel and degrade B20, members

149 of the abundant taxa Byssochlamys and Wickerhamomyces, were isolated. These

150 isolates were used to determine their ability to metabolize B20 biodiesel and spatially

151 induce corrosion of carbon steel.

152 Results

153 Characterization and Phylogenetic Identification of Fungi Isolated from B20

154 Biodiesel

155 The fungal ITS sequences that were sequenced suggested that the filamentous

156 fungal isolate was most closely related to Byssochlamys nivea (Fig. 1A) and the yeast

157 isolate was most closely related to Wickerhamomyces anomalus (Fig. 1B). 


\section{Fungal Growth in Bioreactors}

159 Byssochlamys AF001 and Wickerhamomyces SE3 were presumably in lag phase and

160 had no apparent growth during the first 7 days in the bioreactors. However, after 14

161 days the MPN of Byssochlamys on the surface of the carbon steel witness coupons had

162 increased over an order of magnitude, the density of Wickerhamomyces on the coupon

163 surfaces and the liquid medium had increased in a similar fashion based on CFUs (Fig

$1642)$.

\section{Acidification of Aqueous Medium}

When Byssochlamys sp. AF001 and Wickerhamomyces sp. SE3 were grown on

167 B20 biodiesel as the sole carbon and energy source, the $\mathrm{pH}$ of the aqueous phase did

168 not decrease significantly after 7 days relative to abiotic controls (Fig. 4C). By day 14

169 and 21 both fungal isolates significantly reduced $(\mathrm{p}<0.05)$ the $\mathrm{pH}$ of the medium by two

170 or more orders of magnitude compared to the abiotic controls at those times. The

171 Byssochlamys isolate was responsible for the greatest reduction in $\mathrm{pH}(4.33 \pm 0.31)$ of

172 the aqueous phase after 21 days. The Wickerhamomyces isolate decreased the

173 aqueous $\mathrm{pH}$ to a mean of $5.37 \pm 0.21$ after 21 days.

\section{Direct Measurement of Corrosion}

175 Corrosion rates (MPY) of the carbon steel coupons by each isolate were not 176 significantly different than abiotic controls after 7 and 14 days (Fig 4A). After 21 days,

177 incubations with the Byssochlamys isolate had significantly greater $(p<0.05)$ corrosion 178 rates compared to abiotic controls. Wickerhamomyces SE3 did not produce significantly

179 higher corrosion rates when compared to abiotic controls. The total area pitted of

180 witness coupons was significantly higher than that of abiotic controls only in bioreactors 
181 inoculated with Byssochlamys after 14 and 21 days $(p<0.05)$ (Fig 4B). The greatest pit

182 depth recorded for all Byssochlamys cultures was $132.7 \mu \mathrm{m}$ whereas the maximum pit

183 depth recorded for all Wickerhamomyces SE3 reactors was $90.7 \mu \mathrm{m}$.

184 Colocation of biology and corrosion

In static cultures the $\mathrm{pH}$ of the aqueous phase was significantly lower $(\mathrm{p}<0.05)$

186 for both Byssochlamys and Wickerhamomyces compared to abiotic controls (Fig. 5A).

187 Corrosion rates were lowest in the organic (fuel) phase and elevated in both the

188 aqueous phase (ASW) and at the interface of fuel and medium (Fig. 6B). Corrosion

189 rates in the fuel phase were significantly higher than abiotic controls $(p<0.05)$, six times

190 greater in cultures inoculated with Byssochlamys relative to uninoculated controls. In

191 cultures inoculated with Wickerhamomyces, corrosion rates were not significantly

192 different $(p>0.05)$ from controls in the fuel phase. Corrosion rates at the fuel/medium

193 interface and in the growth medium were significantly higher for both Byssochlamys and

194 Wickerhamomyces relative to abiotic controls $(p<0.05)$. The greatest corrosion rates

195 (2.1 \pm 0.23 MPY Byssochlamys and 1.8 \pm 0.36 MPY Wickerhamomyces) were observed

196 at the fuel/medium interface. Byssochlamys produced more and deeper pitting corrosion

197 at the fuel/medium interface compared to the uninoculated control (Fig. 7).

198 Fuel Degradation

199 Both Byssochlamys and Wickerhamomyces were capable of growth in B20,

200 metabolizing FAME and alkane components of the fuel. As a result, the peak area of all

201 detectable FAME components and alkanes decreased over time in Byssochlamys and

202 Wickerhamomyces cultures (Figure 8). Compared to uninoculated controls, both

203 Byssochlamys and Wickerhamomyces cultures metabolized greater than $50 \%$ of the 
204 cis-9-oleic acid methyl ester by day 21. Both fungi decreased the concentration of Cis-9-

205 Oleic acid methyl ester more than any other fuel component after 21 days (measured as

206 peak area). Analysis of the uninoculated controls over the 21 days showed evaporation

207 of fuel components with approximately $15 \%$ reduction in the components compared to

208 the unexposed fuel.

209 Discussion

210 Complex interactions between microorganisms, fuels, and metallic surfaces in

211 situ make it challenging to directly link the microbial degradation of biodiesel to

212 corrosion (29). Isolation of the relevant microorganisms and controlled laboratory

213 experimentation is critical to linking microbial growth and metabolic activity to the

214 corrosion of infrastructure. Both fungi isolated and identified in this study, Byssochlamys

215 AF004 and Wickerhamomyces SE3, represented some of the most abundant fungi

216 identified in a longitudinal study of microbial contamination and microbiologically

217 influenced corrosion in B20 storage tanks (28). We measured the ability of these

218 organisms to both degrade B20 and accelerate corrosion of carbon steel in controlled

219 laboratory experiments. The co-location of microbial biomass and the greatest amount

220 of corrosion at the interface of fuel and water provided a clear link of where the potential

221 for corrosion would be highest in storage tanks, enhancing our ability to more

222 specifically target regions of fuel tanks for mitigation and increasing the chance of early

223 detection of MIC.

224 Two fungi that were abundant in contaminated B20 fuel samples included a

225 filamentous fungus, Byssochlamys, and a yeast, Wickerhamomyces. Both were capable

226 of growth on B20 as a sole carbon and energy source by degrading the readily 
227 oxidizable FAME components of B20 and several of the alkanes within 21 days.

228 Currently the U.S. DOE and the European Union authorizes the use of B5 and B7

229 respectively to be added to diesel fuels $(30,31)$. Our work suggests that even in fuels

230 with low concentrations of FAME such as B5 or B7 (supposedly 'neat' ULSD) biofouling,

231 microbiologically influenced corrosion, and fuel degradation can become more common

232 as biodiesel is added to petroleum diesel.

233 Biodegradation of both hydrocarbons and FAME in biodiesel blends produces

234 organic acids, which acidifies both the fuel and any aqueous environments, increasing

235 the corrosion of carbon steel $(32,33)$. Both fungal isolates in this study could degrade

236 alkanes and FAME in B20 biodiesel and appeared to degrade cis-9-oleic acid methyl

237 ester and cis-11-eicosonic acid methyl ester more readily than the other compounds.

238 Some studies have demonstrated that both fungi and bacteria can metabolize and

239 incorporate unsaturated fatty acids much more rapidly than saturated fatty acids and

240 has been attributed to linear fatty acids being less efficient in entering inside cells for

241 metabolic processes $(35,36)$.

242 Biofilms can accelerate corrosion rates by creating localized, concentrated acidic

243 conditions at metallic surfaces or by partitioning oxygen, generating oxygen corrosion

244 cells. Both of these mechanisms increase "pitting" or localized corrosion (34). Surface

245 analysis conducted by white light profilometry showed that pitting corrosion was more

246 severe on coupons exposed to the filamentous fungus Byssochlamys AF001 compared

247 to the yeast Wickerhamomyces. This difference could be due to the thick biofilms

248 formed by the filamentous fungus. Other filamentous fungi such as Aspergillus niger

249 form thick biofilms over steel surfaces, resulting in more pitting corrosion (37). After 21 
250 days, Byssochlamys biofilms generated approximately 30 -fold more pitted area than the

251 abiotic controls, and 4 times more than on coupons exposed to the yeast

252 Wickerhamomyces. The elevated corrosion observed in the Byssochlamys cultures

253 confirms previous studies in USAF B20 storage tanks that suggested filamentous fungi

254 contributed to MIC (28).

While we confirmed that both fungi were capable of MIC, materials did exhibit some surface passivation, or reduction in corrosion rates over time. Corrosion rates

257 decreased slightly over the course of the 21-day bioreactor experiment, possibly due to 258 the formation of passivating iron oxides on the surface of the metal. This passivation 259 could prevent further iron oxidation from occurring as rapidly as when neutral iron is 260 exposed to the medium $(38,39)$. Corrosion rates plateaued over time similar to the 261 corrosion dynamics that were observed in situ where Byssochlamys was the most 262 abundant fungal population (40). Although overall corrosion rates decelerated over the 263 course of 12 months, significantly more deep pits were detected, suggesting that long264 term corrosion modeling remains challenging.

265 Both isolated fungi are capable of growth under oxic conditions, and most of the 266 observable fungal and bacterial taxa identified in situ were either facultative anaerobes 267 or known aerobic microorganisms (28). As the fungi grew, an obvious biofilm formed at 268 the fuel:water interface. This growth could provide a physical niche in that the fungi 269 could use the available oxygen in the fuel for metabolism while excluding other 270 microorganisms from that space and define which organisms can grow in fuel storage

271 tanks. Biodiesel and biodiesel blends (such as B20) contain more dissolved oxygen 272 than ultra-low sulfur diesel (41). Aerobic metabolism at the fuel:water interface would 
273 deplete the oxygen present in the immediate environment, including the aqueous

274 phase. Dissolved oxygen in the much larger volume of the fuel would be limited by

275 diffusion into the aqueous phase, which would more than likely remain anoxic when

276 contaminated with metabolizing microorganisms. The resulting oxygen concentration

277 gradient would be most prominent at the interface of fuel and water where the fungi we

278 isolated are actively growing within a fuel tank, generating an oxygenic corrosion cell

279 and leading to exacerbated, aggressive pitting-type corrosion (40, 42).

Although correlative links between microbial degradation of both fossil and bio-

281 based fuels and corrosion are well established, the direct connection between fungal

282 taxa and rates or types of corrosion is less developed (43-45). The controlled

283 experiments in this study link active fungal metabolism to the aerobic degradation of

284 B20, the production of biofilms at the fuel/water interface, and pitting corrosion. Future

285 research will address how potential metabolic interactions between Byssochlamys and

286 the bacterial populations might affect the rates of fuel degradation and corrosion. The

287 FAME components of biodiesel are more amenable to biodegradation than petroleum-

288 based hydrocarbons (Figure 8), suggesting persistent challenges in the long-term

289 storage of biodiesel $(46,47)$. While we tested a $20 \%$ biodiesel blend, biodiesel is also

290 present in ULSD in the U.S. at concentrations up to 5\%; added to compensate for the

291 loss of lubricity in diesel fuel from the removal of organosulfur compounds. Blends in the

292 EU contain up to 7\% biodiesel. Therefore, even in fuels denoted as "neat" or ULSD the

293 degradation of FAME and hydrocarbons represents an increased corrosion risk within

294 storage infrastructure. These findings have identified the challenges associated with the

295 incorporation of FAME in diesel fuels and will inform best management practices to 
296 allow the continued use of renewable fuels, while also reducing the risk of

297 microbiologically influenced corrosion to global energy infrastructure.

298 MATERIALS AND METHODS

299 Isolation, identification, and growth of fungal isolates. Byssochlamys sp. strain

300 AF004 and Wickerhamomyces sp. strain SE3 were isolated from contaminated B20

301 biodiesel storage tanks at an Air Force Base in the southeast United States (32). To

302 isolate the fungi, $1 \mathrm{~L}$ of B20 fuel was passed through a Steritop ${ }^{\mathrm{TM}}$ bottle top filter unit

303 with a $40 \mathrm{~cm}^{2}$ filter area and $0.22 \mu \mathrm{m}$ nominal pore size (Millipore Sigma). This filter was

304 cut out with a sterile scalpel, transferred to a $1.5 \mathrm{~mL}$ Eppendorf tube containing $500 \mu \mathrm{L}$

305 of phosphate buffered saline $(\mathrm{pH} 7.4)$, and the biomass was resuspended by vortexing

306 at maximum speed for $1 \mathrm{~min}$. The biomass suspension was diluted in phosphate

307 buffered saline (pH 7.4), spread onto Hestrin Schramm (HS) agar medium (per L: 20g

308 glucose, $5 \mathrm{~g}$ yeast extract, $5 \mathrm{~g}$ peptone, $2.7 \mathrm{~g} \mathrm{Na} \mathrm{HPO}_{4}, 1.15 \mathrm{~g}$ citric acid, $7.5 \mathrm{~g}$ Agar; $\mathrm{pH}$

309 adjusted to 6.0 with diluted $\mathrm{HCl}$ or $\mathrm{NaOH})(48)$ and incubated at $25^{\circ} \mathrm{C}$ for 7 days.

$310 \quad$ Fungal growth was transferred for isolation repeatedly until pure cultures

311 (repeatedly singular morphologies) were obtained. The fungal isolates were identified by

312 extracting genomic DNA with the Quick-DNA Fungal/Bacterial Miniprep Kit (Zymo

313 Research; Irvine (Fig, CA), amplifying and sequencing the internal transcribed spacer

314 (ITS) region. Specifically, the ITS region for each isolate was amplified with the primers

315 ITS1-F (5'CTTGGTCATTTAGAGGAAGTAA-3') and ITS4 (5'-

316 TCCTCCGCTTATTGATATGC-3') (49, 50)in a PCR using 5 PRIME HotMasterMix

317 (Quanta Biosciences, Beverly, MA, United States). The amplified DNA was purified

318 using ExoSap-IT TM (Thermo Fisher Scientific) according to the manufacturer's 
319 instructions. Once purified, the amplified DNA was submitted for BigDye ${ }^{\circledR}$ Direct

320 Cycle sequencing with each amplification primer on the 3130xI Genetic Analyzer

321 (Biology Core Molecular Laboratory, University of Oklahoma). The resulting sequences

322 for each amplicon were trimmed for quality to only include nucleotides with a Q-score $\geq$

32330 and merged using CAP3 (51). The trimmed and merged sequences were then

324 submitted to NCBI to identify their closest neighbor using MegaBLAST. The MEGA X

325 software package (52) was used to build a maximum likelihood tree (Tamura-Nei model,

326 bootstrapped with 500 samplings) containing sequences from the isolates and closest

327 neighbors (Fig. 1).

328 A spore suspension of the filamentous fungus Byssochlamys sp. was used as

329 the normalized inoculum in the experiments described below. To prepare the spore

330 suspension, $4 \mathrm{~mL}$ of PBS was added to the surface of HS agar containing hyphal

331 growth of Byssochlamys sp., and an inoculating loop was used to scrape off the fungal

332 growth. The PBS solution was then collected from the plate and filtered through a $10 \mu \mathrm{m}$

333 pore size nitrocellulose filter, to separate spores from hyphal biomass. The spores were

334 then centrifuged at $10,000 \times$ RCF for 1 minute. The supernatant was decanted, and

335 sterilized PBS was added back to the spore pellet and vortexed to resuspend the

336 spores in solution. This was repeated for a total of three washes. Spore concentrations

337 were determined using a Petroff-Hausser counting chamber then diluted to adjust the

338 inoculum concentration to $1 \times 10^{4}$ spores $/ \mathrm{mL}$.

339 To produce a suspension of yeast cells, Wickerhamomyces sp. was grown in HS

340 broth for 48 hours and was centrifuged at $10,000 \times$ RCF to pellet cell mass. The

341 supernatant was decanted, and sterilized PBS was added to the cell pellet. This pellet 
342 was centrifuged at $10,000 \times$ RCF for 1 minute. The supernatant was decanted, and

343 more sterilized PBS was added to the cell pellet and vortexed to resuspend the cells in

344 suspension. This was repeated for a total of three washes. Cell concentrations were

345 determined using a Petroff-Hausser counting chamber and diluted to adjust the 346 inoculum concentration to $1 \times 10^{4}$ cells $/ \mathrm{mL}$.

Quantification and Characterization of B20 Biodiesel Fuel Biodegradation.

348 Fungal biodegradation of B20 was evaluated by direct measure of fungal growth and

349 consumption of the fuel compounds. Fungal growth and ability to degrade B20 biodiesel

350 was measured every 7 days for 21 days. All biodegradation experiments were

351 incubated aerobically at $25^{\circ} \mathrm{C}$ and non-shaking (i.e. static) conditions. Test tubes (16 x

$352150 \mathrm{~mm}$ ) were filled with $5 \mathrm{~mL}$ filter sterilized B20 as the sole carbon and energy source

353 and ASW liquid medium in a 1:10 fuel to ASW ratio. Wickerhamomyces and

354 Byssochlamys were initially grown on HS broth and spun down at $10,000 \times$ RCF to

355 pellet cell mass. This pellet was resuspended in PBS by vortex, centrifuged and

356 resuspended a total of 3 times to remove any potential nutrient carry over before

357 inoculation. Finally, the cell/spore density was determined using a hemocytometer,

358 allowing the B20 and ASW to be inoculated to a final concentration of $1 \times 10^{4}$ cells $/ \mathrm{mL}$ or

359 spores $/ \mathrm{mL}$ respectfully. The fuel phase of these cultures was separated using a $10 \mathrm{~mL}$

360 separatory funnel and diluted 1:10 in hexane $\geq 97.0 \%$ (GC) and analyzed by Gas

361 Chromatography/Mass Spectrometry (GC/MS). The chemical composition of the B20

362 biodiesel samples prior to degradation was determined by GC/MS using a Shimadzu

363 QP 2010 SE (Shimadzu Corporation, USA). Each sample was diluted 1:10 with hexane

364 prior to injection. A volume of $1 \mu \mathrm{L}$ was injected via autosampler with a split ratio of $1: 10$ 
365 for a final dilution of $1: 100$. Injection started at $300^{\circ} \mathrm{C}$, the oven was at $40^{\circ} \mathrm{C}$ with a 1.5

$366 \min$ hold, which increased to $320^{\circ} \mathrm{C}$ at a rate of $10^{\circ} \mathrm{C} \mathrm{min}{ }^{-1}$. Chemical components were

367 separated with a Restek Column Rxi 5Sil with dimensions: $30 \mathrm{~m}, 0.25 \mathrm{~mm}$ ID, $0.25 \mu \mathrm{m}$.

368 High purity helium was used as a carrier gas at a linear velocity of $36.8 \mathrm{~cm} \mathrm{~s}^{-1}$. Mass

369 spectra were analyzed in scan mode with the following parameters: interface at $320^{\circ} \mathrm{C}$,

370 ion source $200^{\circ} \mathrm{C}$, solvent cut of $2.75 \mathrm{~min}$, event time of $0.25 \mathrm{sec}$ and scan speed of

371 2000. Each Total Ion Chromatogram (TIC) was processed using the software

372 LabSolutions version 4.20 (Shimadzu Corporation, USA). Peaks were identified using

373 the mass spectra library NIST version 14 and verified and quantified using reference

374 standards for FAME (Supelcoß 37 Component FAME Mix, Sigma Aldrich, USA) and

375 saturated alkanes (C7-C40 Saturated Alkanes Standard, Sigma Aldrich, USA). Major

376 alkane and FAME peaks were identified by the NIST library replicates and underwent

377 destructive sampling of quintuplicates at each time point. The degradation of fuel

378 compounds was measured by determining the amount (\%) of the remaining fuel

379 components relative to a non-exposed control. For all experiments, negative controls

380 (un-inoculated) were included to evaluate contamination risks and assess abiotic

381 degradation. Un-amended controls (inoculated but with no B20) were also included to

382 evaluate nutrient carryover from the initial inocula.

384 Corrosion in Bioreactors. Three CDC biofilm bioreactors ${ }^{\circledR}$ (BioSurface Technologies

385 Corp.) were filled with $3 \mathrm{~mL}$ of B20 biodiesel and $297 \mathrm{~mL}$ of Artificial Sump Water (ASW,

386 per L: $0.015 \mathrm{~g} \mathrm{NaCl}, 0.035 \mathrm{~g} \mathrm{NaF}, 0.02 \mathrm{~g} \mathrm{CaCl}_{2}, 0.018 \mathrm{gNO}_{3}, 0.01 \mathrm{~g} \mathrm{Na}_{2} \mathrm{SO}_{4}, 0.015 \mathrm{~g}$

$387\left(\mathrm{NH}_{4}\right)_{2} \mathrm{SO}_{4}$, and $\left.0.017 \mathrm{~K} \mathrm{~K}_{2} \mathrm{HPO}_{4}\right)(1: 100$ ratio of $\mathrm{B} 20: \mathrm{ASW})$ and inoculated to a final 
concentration of $1 \times 10^{4}$ Byssochlamys spores $/ \mathrm{mL}$ or Wickerhamomyces cells $/ \mathrm{mL}$. Grade 1018 carbon steel circular disk coupons $(1.27 \mathrm{~cm}$ diameter x $3.82 \mathrm{~mm}$ thick; BioSurface Technologies Corp., Bozeman, MT) were washed in acetone to remove any machine oil, weighed to obtain initial mass, and sterilized prior to use by exposing them to UV

392 light for 15 minutes on each side. In an AirClean 600 PCR Workstation (AirClean

393 Systems Inc., Creedmoor, NC), three carbon steel disks were inserted into each of

394 three polypropylene coupon holders. Three of the coupon holders were inserted into

395 each of the three bioreactors. The bioreactors were sampled weekly for three weeks by

396 removing one of the polypropylene coupon holders and replacing it with a sterilized

397 bioreactor blank coupon holder, allowing for three technical and three biological

398 replicates. The coupons collected from each reactor were cleaned and weighed to

399 determine mass loss, which was used to calculate corrosion rates (described below).

400 The surface topology of each coupon was also determined to quantify the area and

401 depth of any corrosion. The $\mathrm{pH}$ of the aqueous phase was determined at each sampling

402 time, as was the viability of the isolates or sterility of controls. Viability measurements of

403 the planktonic microorganisms were made by sampling medium from the bioreactors

404 and conducting MPN/mL measurements for Byssochlamys AF001 and CFU/mL

405 measurements for Wickerhamomyces SE3 using liquid or solid HS medium,

406 accordingly. The population density of microorganisms attached to each coupon was

407 determined by removing biomass with a sterile nylon swab and transferring it to sterile

408 PBS. The biomass was suspended by vortexing and used to determine MPN or

$409 \mathrm{CFU} / \mathrm{mL}$ as described above. After removing the biomass with a swab, each coupon

410 was cleaned using ASTM method G01-03 C3.5 and immediately weighed to determine 
411 mass loss. After being weighed, the coupons were stored in an anaerobic chamber to

412 prevent further abiotic oxidation until surface analysis could be conducted. The technical

413 replicates were averaged and used for data analysis.

$414 \quad$ The surface of each coupon was analyzed using white light profilometry

415 (Nanovea PS50; Nanovea, Inc.; Irvine, CA). To ensure that the same area was being

416 scanned on all coupons, a clip was used to align the coupons in the same location on

417 the profilometer's stage (Supplemental information contains 3D files that can be 3D

418 printed and examined).

419 A $6 \times 6 \mathrm{~mm}$ area was scanned on the coupon and visualized using Mountains

420 Software version 6.3 (Digital Surf). Intensity and height maps were combined in the

421 software and leveled using a least squares plane method by subtraction. Non-measured

422 points were filled in using calculations of points from the nearest neighbors. Pits were

423 defined as any points that were greater than $20 \mu \mathrm{m}$ below the mean surface average.

424 Maximum pit depth and total pitted area were calculated from the surface analyses.

425 Localizing Corrosion in Fuel, Interphase, and Aqueous Phases. Static

426 corrosion experiments were set up with the intent of determining where corrosion was

427 greatest, in the fuel, interphase, or aqueous phase of water-containing fuel systems.

428 The static incubations were made by first filling test tubes $(16 \times 150 \mathrm{~mm})$ with $3 \mathrm{~mL}$ of

429 sterile ASW medium to mimic water present in the underground storage tanks from

430 runoff or condensation within the tank (53). B20 biodiesel was filter sterilized into an

431 autoclaved 1L Schott Bottle using a Steritop ${ }^{\mathrm{TM}}$ bottle top filter unit with a $40 \mathrm{~cm}^{2}$ filter

432 area and $0.22 \mu \mathrm{m}$ nominal pore size (Millipore Sigma) and $3 \mathrm{~mL}$ of this biodiesel was

433 aseptically added to the tubes, resulting in a 1:1 fuel to ASW ratio. Non-galvanized 
434 carbon steel brads $(0.5 \mathrm{~mm}$ diameter $\times 19 \mathrm{~mm}$ length) were washed in acetone to

435 remove any machine oil, weighed to obtain initial mass, and autoclaved anaerobically in

436 Balch tubes with a $\mathrm{N}_{2}$ headspace to prevent abiotic corrosion and ensure sterility prior

437 to the experiment. The brads were inserted into holes drilled into nylon bolts at three

438 levels before the autoclave cycle. The holes were positioned so that the brads were

439 exposed to either the fuel phase, the fuel-water interface, or the aqueous phase

440 (Supplemental Figure 1).

$441 \quad$ Five replicates of the static corrosion tubes described above were either

442 inoculated with a final concentration of $1 \times 10^{4}$ Byssochlamys sp. spores or

443 Wickerhamomyces sp. cells per $\mathrm{mL}$. An additional five tubes remained uninoculated as

444 controls. The tubes were incubated for 21 days at room temperature in the dark. After

44521 days, the $\mathrm{pH}$ of the aqueous phase was measured using an Oakton $\mathrm{pH}$ Spear

446 waterproof Pocket $\mathrm{pH}$ Testr ${ }^{\mathrm{TM}}$. The viability of the organisms (or sterility of the controls)

447 was assessed by growth on HS agar medium and direct microscopy. Viability of the

448 Wickerhamomyces sp. was determined by CFU/mL on HS agar medium. Growth of the

449 Byssochlamys sp. was confirmed by observation of hyphal growth under light

450 microscopy by preparing a wet mount and examining at 100x magnification. Corrosion

451 rates were calculated from mass loss using the approach described below (Calculating

452 corrosion rates from mass loss measurements).

453 Additional static corrosion experiments were conducted with flat coupons that

454 traversed the fuel, interphase, and aqueous phases in order to visualize the surface of

455 the coupon across all phases with scanning electron microscopy and measure the area

456 and depth of corrosion pits using the white light profilometry as described above. The 
457 coupons used for this experiment were composed of 1018 carbon steel, 3" inches long,

458 3/8" inches wide, and 1/16" inches thick, and finished by the manufacturer by sanding

459 using a 120-grit belt (Alabama Specialty Products, Inc.; Munford, AL). Each coupon was

460 placed in separate $250 \mathrm{~mL}$ Erlenmeyer flasks containing $25 \mathrm{~mL}$ of sterile B20 biodiesel

461 and ASW for a 1:1 ratio. These flasks were either inoculated with $1 \times 10^{4}$ spores of the

462 filamentous fungus Byssochlamys or kept uninoculated as controls, incubated at $25^{\circ} \mathrm{C}$

463 for 90 days, and used to quantify and characterize surface corrosion.

464 The surface of the coupons was analyzed using white light profilometry as

465 described above. To ensure that the same area $20 \times 8 \mathrm{~mm}$ area was being scanned on

466 all coupons, a clip was used to align the coupons in the same location on the

467 profilometer's stage (Supplemental Information). subtraction. Within the software, non-measured points were filled in using calculations

471 of points from the nearest neighbors. Pits were defined as any points that were less

472 than $20 \mu \mathrm{m}$ below the mean surface average. Maximum pit depth and total pitted area

473 were calculated from the surface analyses.

$474 \quad$ Calculation of corrosion rates from mass loss measurements. Following

475 incubation, carbon steel brads and coupons were cleaned using ASTM method G01-03

476 C3.5 (54). The final mass was measured and used to determine the rates of corrosion in

477 milli-inches per year (MPY) using the following equation:

$478 \quad$ Corrosion rate $=\frac{K * W}{(A * T * D)}$ where

$479 \quad \mathrm{~K}=$ Mils per year (MPY) rate 
$\mathrm{T}=$ Time of exposure in hours

$$
A=\text { Area in } \mathrm{cm}^{2}
$$

$\mathrm{W}=$ Mass loss in grams (Initial coupon mass - final coupon mass)

$\mathrm{D}=$ Density in $\mathrm{g} / \mathrm{cm}^{3}$

Statistical analysis and data visualization. Statistical analysis and figure

485 generation was carried out in R version 3.3.3 and GraphPad Prism 8.3.0. Significant

486 differences were calculated using two-way ANOVA with a Tukey's HSD to determine

487 significant differences in corrosion rates, maximum pit depths, total pitted areas, and $\mathrm{pH}$

488 between the isolates and uninoculated controls.

\section{ACKNOWLEDGMENTS}

We acknowledge the men and women of the US Air Force and Civilian Personnel

491 at US Air Force bases; their cooperation and assistance were critical to this research.

492 Additionally, we would like to thank Emily Junkins for insightful comments during the

493 development of this manuscript. This work was supported by the Air Force Research

494 Laboratory Biological Materials and Processing Research Team, Materials and

495 Manufacturing Directorate and the U.S. Department of Defense Office of Corrosion

496 Policy \& Oversight Technical Corrosion Collaboration (Grant \# FA7000-15-2-0001).

\section{Works Cited}

500 1. Singleton L, Weller CE. 2001. Prosperity wasn't just around the corner. Economic Policy

501 Institute 166. 
2. John V. 2001. Economic impact of the terrorist attacks of September 11, 2001. Atl Econ J

$503 \quad 29: 353-357$.

504 3. U.S. Energy Information Administration. 2020. biofuels explained use of biomass-based diesel 505 fuel.

4. U.S. Energy Information Administration. 2019. Biodiesel and other renewable fuels overview.

5082019 and biomass based diesel volume for 2020. 83 FR 63704

6. Ogunkunle O, Noor AA. 2019. A review of global current scenario of biodiesel adoption and

510 combustion in vehicular diesel engines. Energy Reports 5:1560-1579.

511 7. Department of Defense. 2016. Department of Defense 2016 operational energy strategy.

512 8. Özener O, Yüksek L, Ergenç AT, Özkan Muammer. 2014. Effects of soybean biodiesel on a

513 DI diesel engine performance, emission and combustion characteristics. Fuel 115:875-883.

514 9. Meher LC, Sagar DV, Naik SN. 2006. Technical aspects of biodiesel production by

515 transesterification — a review. Renewable and Sustainable Energy Reviews 10:248-268

516 10. Zuleta EC, Baena L, Rios LA, Calderón JA. 2012. The oxidative stability of biodiesel and its

517 impact on the deterioration of metallic and polymeric materials: a review. J Braz Chem Soc

$518 \quad 23: 2159-2175$.

519 11. Demirbaş A. 2008. Biodegradability of biodiesel and petrodiesel fuels. Energy Sources, Part

520 A: Recovery, Utilization, and Environmental Effects. 31:169-174 
521 12. Prince RC, Haitmanek C, Lee CC. 2008. The primary aerobic biodegradation of biodiesel

522 B20. Chemosphere 71:1446-1451.

523 13. Aktas DF, Lee JS, Little BJ, Ray RI, Davidova IA, Lyles CN, Suflita JM. 2010. Anaerobic

524 metabolism of biodiesel and its impact on metal corrosion. Energy Fuels 24:2924-2928.

525 14. Thomas AO, Leahy MC, Smith JWN, Spence MJ. 2017. Natural attenuation of fatty acid

526 methyl esters (FAME) in soil and groundwater. Quarterly Journal of Engineering Geology and

527 Hydrogeology 50:301-317.

528 15. Houten SM, Wanders RJA. 2010. A general introduction to the biochemistry of

529 mitochondrial fatty acid $\beta$-oxidation. J Inherit Metab Dis 33:469-477.

530 16. Morawe M, Hoeke H, Wissenbach DK, Lentendu G, Wubet T, Kröber E, Kolb S. 2017.

531 Acidotolerant bacteria and fungi as a sink of methanol-derived carbon in a deciduous forest soil.

532 Front Microbiol 8:1361.

533 17. Abbasian F, Lockington R, Mallavarapu M, Naidu R. 2015. A comprehensive review of

534 aliphatic hydrocarbon biodegradation by bacteria. Appl Biochem Biotech 176:670-699.

535 18. Colombo JC, Cabello M, Arambarri AM. 1996. Biodegradation of aliphatic and aromatic

536 hydrocarbons by natural soil microflora and pure cultures of imperfect and lignolitic fungi.

537 Environ Pollut 94:355-362.

538 19. Rojo F. 2010. Enzymes for aerobic degradation of alkanes, p 781-797. In Timmis KN (eds),

539 Handbook of hydrocarbon and lipid microbiology, Springer, Berlin, Heidelberg. 
540 20. Skovhus T, Eckert RB, Rodrigues E. 2017. Management and control of microbiologically

541 influenced corrosion (MIC) in the oil and gas industry-Overview and a North Sea case study. J

542 Biotechnol 256:31-45.

543 21. Amy PS, Jones DA. 2002. A thermodynamic interpretation of microbiologically influenced 544 corrosion. Corrosion 58:638-645.

545 22. Stewart PS, Franklin MJ. 2008. Physiological heterogeneity in biofilms. Nat Rev Microbiol 546 6:199-210.

547 23. Usher KM, Kaksonen AH, Cole I, Marney D. 2014. Critical review: microbially influenced 548 corrosion of buried carbon steel pipes. Int Biodeterior Biodegradation 93:84-106.

549 24. Bento FM, Beech IB, Gaylarde CC, Englert GE, Muller IL. 2005. Degradation and corrosive 550 activities of fungi in a diesel-mild steel-aqueous system. World J Microbiol Biotechnol 21:135$551 \quad 142$.

552 25. Bücker F, Santestevan NA, Roesch LF, Jacques RJS, Peralba MCR, Camargo FAOC, Bento 553 FM. 2011. Impact of biodiesel on biodeterioration of stored Brazilian diesel oil. Int Biodeterior 554 Biodegradation 65:172-178.

555 26. Mohanan S, Rajasekar A, Muthukumar N, Maruthamuthu S, Palaniswamy N. 2005. The role 556 of fungi on diesel degradation, and their influence on corrosion of API 5LX steel. Corrosion 557 Prevention and Control 4:123-130.

558 27. United States Air Force. Air Force Energy Plan 2010. 
28. Stamps BW, Bojanowski CL, Drake CA, Nunn HS, Lloyd PF, Floyd JG, Emmerich KA,

560 Neal AR, Crookes-Goodson WJ, Stevenson BS, Stamps BW, Bojanowski CL, Drake CA, Nunn

561 HS, Lloyd PF, Floyd JG, Emmerich KA, Neal AR, Crookes-Goodson WJ, Stevenson BS. 2020.

562 In situ linkage of fungal and bacterial proliferation to microbiologically influenced corrosion in

563 B20 biodiesel storage tanks. Front Microbiol 11:167.

564 29. Suflita JM, Lyles CN, Aktas DF, Sunner J. 2014. Biocorrosion issues associated with the use

565 of ultra-low sulfur diesel and biofuel blends in the energy infrastructure, p 313-328. In Liengen

566 T, Basseguy R, Féron D, Birrien V, Understanding biocorrosion: fundamentals and applications,

567 Netherlands, Elsevier Science.

568 30. American Society for Testing and Materials. Standard specification for diesel fuel oils.

569 ASTM International.

570 31. European Union for Standardization. 2009. Automotive fuels - Diesel - Requirements and

571 test methods. EN 590.

572 32. Andrade O. 2016. Master Thesis. Characterization of fungal contaminants in B20 biodiesel

573 storage tanks and their effect on fuel composition. University of Oklahoma, Norman, OK.

574 33. Liebenberg JJ, Skinner W. 1993. Corrosion characteristics of carbon steel in organic solvent

575 containing fatty acids. British Corrosion Journal 2:130-132.

576 34. Little BJ, Lee JS. 2014. Microbiologically influenced corrosion: an update. International

577 Materials Reviews 59:384-393. 
578 35. Papanikolaou S, Chevalot I, Komaitis M, Aggelis G, Marc I. 2001. Kinetic profile of the

579 cellular lipid composition in an oleaginous Yarrowia lipolytica capable of producing a cocoa-

580 butter substitute from industrial fats. Antonie Van Leeuwenhoek 80:215-224.

581 36. Tzirita M, Papanikolaou S, Chatzifragkou A, Quilty B. 2018. Waste fat biodegradation and

582 biomodification by Yarrowia lipolytica and a bacterial consortium composed of Bacillus spp.

583 and Pseudomonas putida. Eng Life Sci 18:932-942.

584 37. Szatmari I, Tudosie L, Cojocaru A, Lingvay M, Prioteasa P, Vişan T. 2015. Studies on

585 biocorrosion of stainless steel and copper in Czapek Dox medium with Aspergillus niger

586 filamentous fungus. UPB Scientific Bulletin, Series B: Chemistry and Materials Science 77:91-

587102.

588 38. Kruger J. 1989. The nature of the passive film on iron and ferrous alloys. Corrosion Science

589 29:149-162.

590 39. Rahner D. 1996. Fe3O4 as part of the passive layer on iron. Solid State Ion 86-88:865-871.

591 40. Little BJ, Lee JS, Ray RI. The influence of marine biofilms on corrosion: a concise review.

592 Electrochim Acta 54:2-7.

593 41. Speidel H, Lightner R, Ahmed I. 2000. Biodegradability of new engineered fuels compared

594 to conventional petroleum fuels and alternative fuels in current use. Appl Biochem Biotechnol

595 84-86:879-897.

596 42. Videla H, Herrera LK. 2005. Microbiologically influenced corrosion: looking to the future.

597 Int Microbiol 8:169-80. 
43. Lee JS, Ray RI, Little BJ. 2010. An assessment of alternative diesel fuels: microbiological

599 contamination and corrosion under storage conditions. Biofouling 26:623-635.

600 44. Kaul S, Saxena RC, Kumar A, Negi MS, Bhatnagar AK, Goyal HB, Gupta AK. 2007.

601 Corrosion behavior of biodiesel from seed oils of Indian origin on diesel engine parts. Fuel

602 Processing Technology 88:303-307.

603 45. Meira M, Santana PB, Araújo AS, Silva CL, Filho J, Ferreira HT. 2014. Oxidative

604 degradation and corrosiveness of biodiesel. Corrosion Reviews 32:143-161.

605 46. Cavalcanti E de, Zimmer A, Bento F, Ferrão M. 2019. Chemical and microbial storage

606 stability studies and shelf life determinations of commercial Brazilian biodiesels stored in carbon

607 steel containers in subtropical conditions. Fuel 993-1007.

608 47. Meyer DD, Beker SA, Bücker F, Peralba MCRP, Frazzon APG, Osti JF, Andreazza R,

609 Camargo FAO, Bento FM. 2014. Bioremediation strategies for diesel and biodiesel in oxisol

610 from southern Brazil. Int Biodeterior Biodegradation 95:356-363.

611 48. Hestrin S, Schramm M. 1954. Synthesis of cellulose by Acetobacter xylinum. 2. Preparation

612 of freeze-dried cells capable of polymerizing glucose to cellulose. Biochem J 58:345-352.

613 49. White TJ, Bruns T, Lee S, Taylor J. 1990. Amplification and direct sequencing of fungal

614 ribosomal RNA genes for phylogenics, p 315-322. In PCR Protocols A Guide to Methods and

615 Applications, Academic Press.

616 50. Gardes M, Bruns TD. 1993. ITS primers with enhanced specificity for basidiomycetes-

617 application to the identification of mycorrhizae and rusts. Mol Ecol 2:113-118. 
618 51. Huang X, Madan A. 1999. CAP3: A DNA sequence assembly program. Genome Res 9:868-

619877.

620 52. Kumar S, Nei M, Dudley J, Tamura K. 2008. MEGA: a biologist-centric software for

621 evolutionary analysis of DNA and protein sequences. Brief Bioinform 9:299-306.

622 53. McNamara CJ, Thomas PD, Leard R, Bearce K, Dante J, Mitchell R. 2006. Corrosion of

623 aluminum alloy 2024 by microorganisms isolated from aircraft fuel tanks. Biofouling 21:257-

624265.

625 54. American Society for Testing and Materials. 2011. Practice for preparing, cleaning, and 626 evaluating corrosion test specimens. ASTM International. 


\section{Figure Captions}

640 FIG 1: Maximum likelihood tree based on ITS sequence similarity among close

641 phylogenetic relatives (NCBI accession numbers in parentheses) of the Byssochlamys

642 sp. AF001 (A) and Wickerhamomyces SE3 (B) isolates. Bootstrap values above 50

643 percent for 500 samples are shown at relevant nodes.

644 FIG 2. Density of planktonic populations (circles) and biofilms (squares) in bioreactors

645 inoculated with Byssochlamys AF001 (MPNs; Black) or Wickerhamomyces SE3 (CFUs;

646 Gray). Error bars represent 95\% confidence intervals for mean Byssochlamys AF001

647 MPNs or Wickerhamomyces SE3 CFUs $(n=3)$.

648 FIG 3. pH values of the aqueous phase in bioreactors inoculated with Byssochlamys

649 AF001(Blue), Wickerhamomyces (Green), or controls (Tan) over time. Bold dashed

650 lines represent the median while the nonbold dashed lines represent the data quartiles.

651 Asterisks indicate a significant difference between the $\mathrm{pH}$ of inoculated and

652 uninoculated controls.

653 FIG 4. A. Corrosion rates in milliinches per year (MPY) of carbon steel coupons in

654 bioreactors inoculated with Byssochlamys (Blue), Wickerhamomyces (Green), and

655 uninoculated controls (Tan) over time. The bold dashed line represents the median

$656(n=3)$ and nonbold dashed lines represent the data quartiles. Asterisks indicate

657 significant difference between the inoculated and abiotic conditions for each time point.

658 B. Total pitted area on carbon steel coupons from bioreactors inoculated with

659 Byssochlamys (Blue), Wickerhamomyces (Green), and uninoculated controls (Tan). The

660 bold dashed line represents the median $(n=3)$ and nonbold dashed lines represent the

661 data quartiles. Asterisks indicate significant difference between the inoculated and 
662 abiotic conditions for each time point. C. Maximum pit depths on carbon steel coupons

663 in uninoculated bioreactors (Tan) and bioreactors inoculated with Byssochlamys (Blue)

664 and Wickerhamomyces (Green) over time.

665 FIG 5. A. pH values of the aqueous phases after incubation of carbon steel brads with 666 Byssochlamys (Blue), Wickerhamomyces (Green) and uninoculated controls (Tan). The

667 bold lines represent the median and the nonbold lines on the plots represent the data 668 quartiles $(n=5)$. Asterisks indicate a significant difference between the inoculated and

669 uninoculated conditions. B. Corrosion rates of carbon steel brads in the organic phases,

670 organic-aqueous interface, and aqueous phase after exposure to the fungal isolates.

671 The bold lines represent the median and the nonbold lines on the plots represent the

672 data quartiles $(n=5)$. Asterisks indicate a significant difference between the inoculated

673 and uninoculated conditions.

674 FIG 6. A. Total pitted area on carbon steel coupons (pits $>20 \mu m$ below mean surface

675 average) inoculated with Byssochlamys (Blue) compared to uninoculated controls (Tan).

676 The bold line represents the medium of the data and the nonbold lines represent the

677 data quartiles $(n=3)$. B. Maximum pit depths on carbon steel coupons in abiotic controls

678 (Tan) and flasks inoculated with Byssochlamys (Blue) over 90 days. The bold line

679 represents the median of the data and the nonbold lines represent the data quartiles

$680(n=3)$

681 FIG 7. Surface depth profiles of carbon steel coupons exposed to the filamentous

682 fungus Byssochlamys and uninoculated controls after 90 days. Biological replicates are

683 show next to each other and depth was calculated from the highest point on the surface

684 of the coupon representing $0 \mu \mathrm{m}$. The color scale bar represents depths in microns. The 
685 dashed bar represents where the interface was located on the coupons with the fuel

686 phase above and the aqeous phase below.

687 FIG 8. Degradation of alkanes and FAME in B20 biodiesel by Wickerhamomyces and

688 Byssochlamys after 7, 14 and 21 days of incubation. The scale bar represents the

689 percent of remaining compound compared to the unexposed control with white

690 indicating no degradation and black indicating complete degradation. 


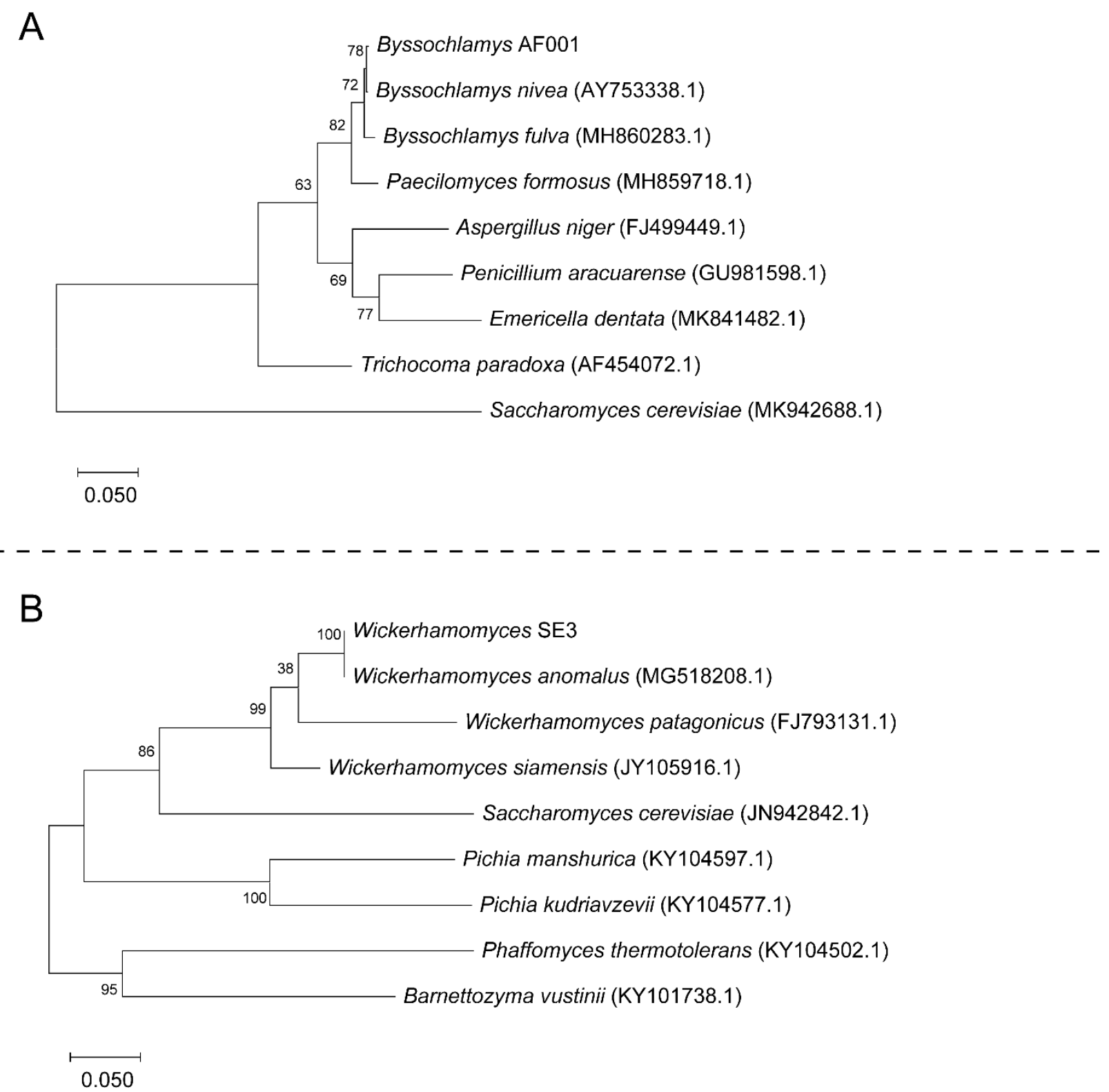

FIG 1: Maximum likelihood tree based on ITS sequence similarity among close phylogenetic relatives (NCBI accession numbers in parentheses) of the Byssochlamys sp. AF001 (A) and Wickerhamomyces SE3 (B) isolates. Bootstrap values above 50 percent for 500 samples are shown at relevant nodes. 


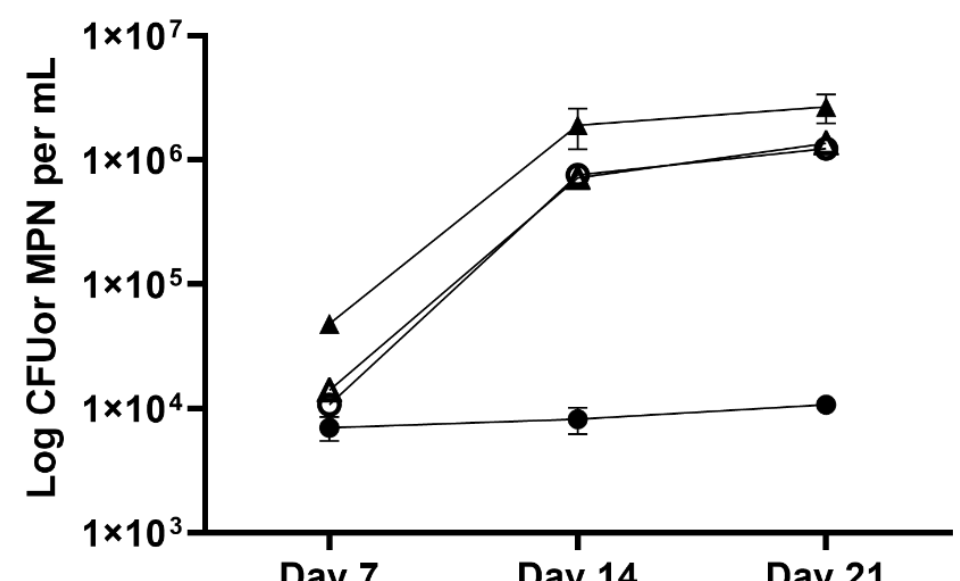

- Byssochlamys Planktonic

- Byssochlamys Surface

- Wickerhamomyces

Planktonic

$\triangle$ Wickerhamomyces Surface

FIG 2. Density of planktonic populations (circles) and biofilms (squares) in bioreactors inoculated with Byssochlamys AF001 (MPNs; Black) or Wickerhamomyces SE3 (CFUs; Gray). Error bars represent 95\% confidence intervals for mean Byssochlamys AF001 MPNs or Wickerhamomyces SE3 CFUs $(n=3)$. 


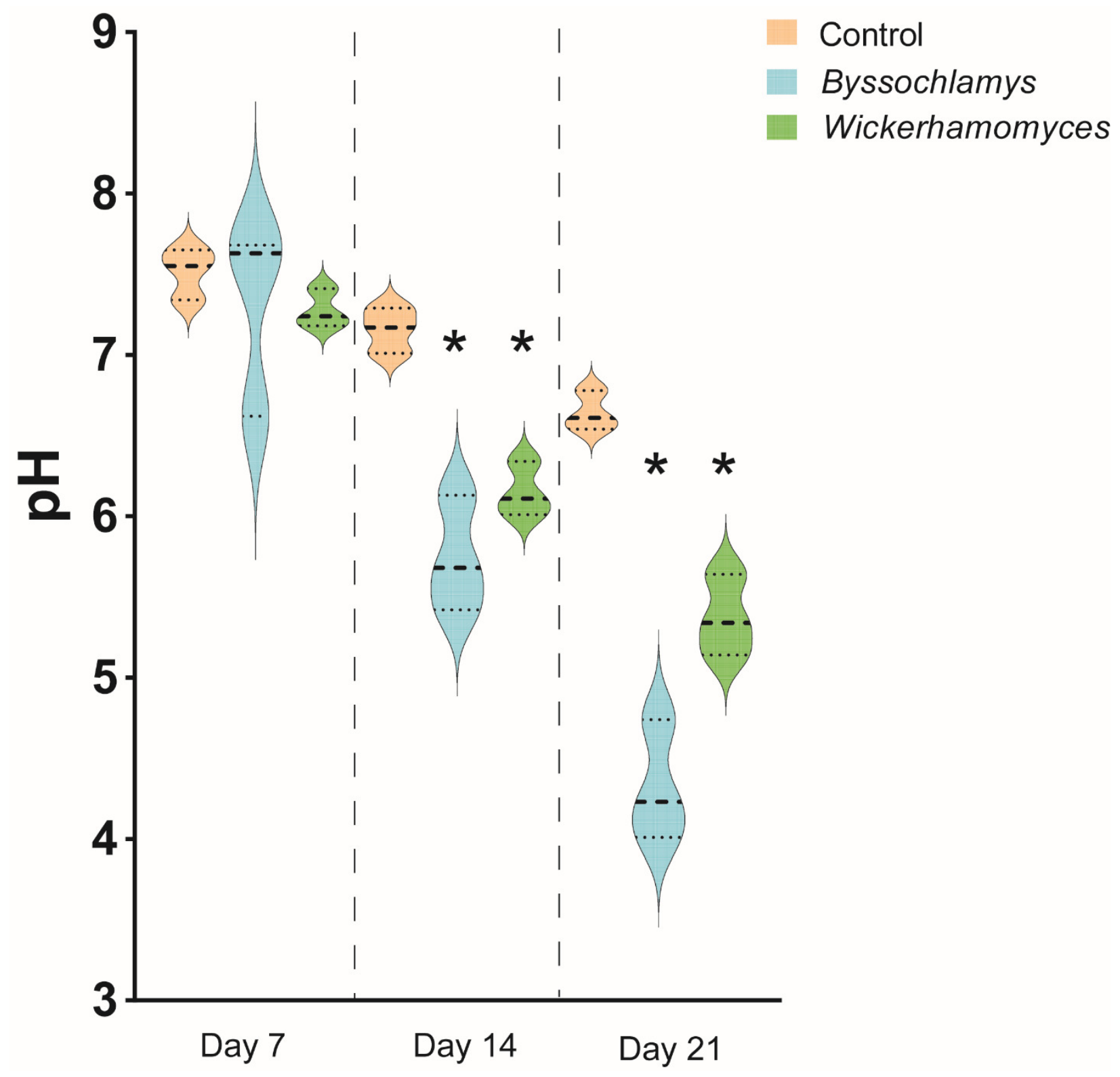

FIG 3. pH values of the aqueous phase in bioreactors inoculated with Byssochlamys AF001(Blue), Wickerhamomyces (Green), or controls (Tan) over time. Bold dashed lines represent the median while the nonbold dashed lines represent the data quartiles. Asterisks indicate a significant difference between the $\mathrm{pH}$ of inoculated and uninoculated controls. 
A

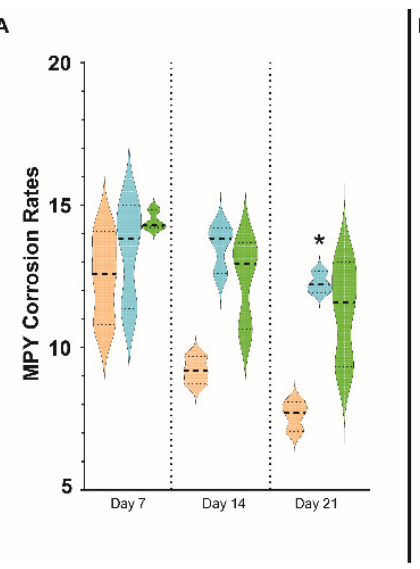

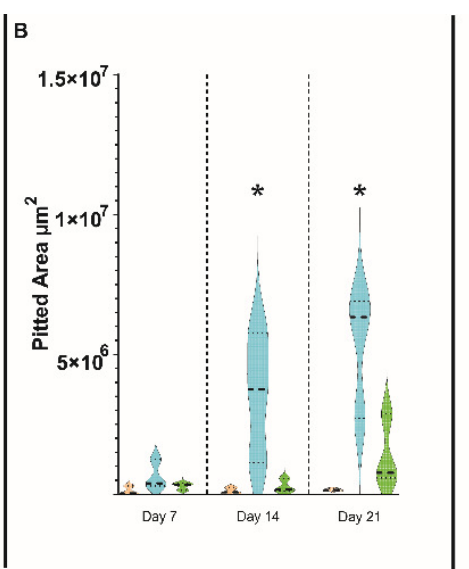

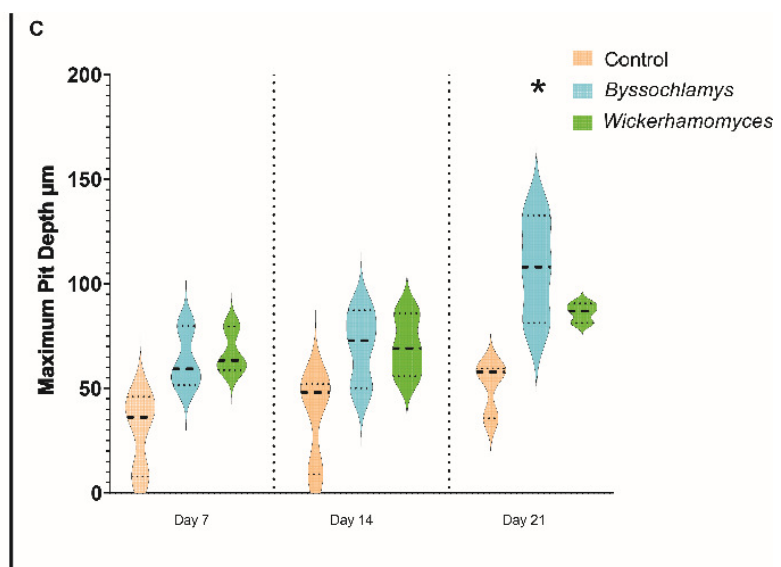

FIG 4. A. Corrosion rates in milliinches per year (MPY) of carbon steel coupons in bioreactors inoculated with Byssochlamys (Blue), Wickerhamomyces (Green), and uninoculated controls (Tan) over time. The bold dashed line represents the median $(n=3)$ and nonbold dashed lines represent the data quartiles. Asterisks indicate significant difference between the inoculated and abiotic conditions for each time point. B. Total pitted area on carbon steel coupons from bioreactors inoculated with Byssochlamys (Blue), Wickerhamomyces (Green), and uninoculated controls (Tan). The bold dashed line represents the median $(n=3)$ and nonbold dashed lines represent the data quartiles. Asterisks indicate significant difference between the inoculated and abiotic conditions for each time point. C. Maximum pit depths on carbon steel coupons in uninoculated bioreactors (Tan) and bioreactors inoculated with Byssochlamys (Blue) and Wickerhamomyces (Green) over time. 

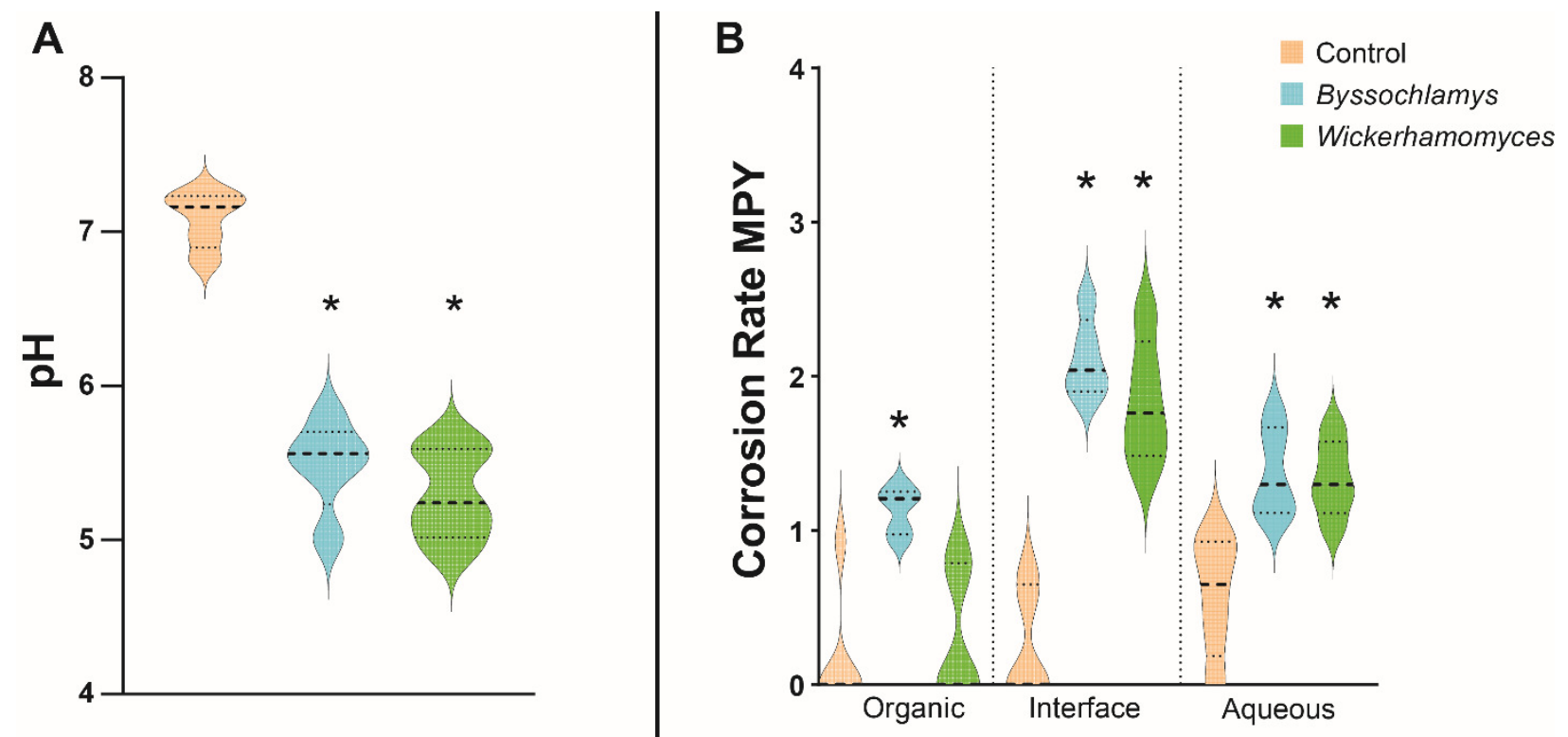

FIG 5. A. pH values of the aqueous phases after incubation of carbon steel brads with Byssochlamys (Blue), Wickerhamomyces (Green) and uninoculated controls (Tan). The bold lines represent the median and the nonbold lines on the plots represent the data quartiles $(n=5)$. Asterisks indicate a significant difference between the inoculated and uninoculated conditions. B. Corrosion rates of carbon steel brads in the organic phases, organic-aqueous interface, and aqueous phase after exposure to the fungal isolates. The bold lines represent the median and the nonbold lines on the plots represent the data quartiles $(n=5)$. Asterisks indicate a significant difference between the inoculated and uninoculated conditions. 
A

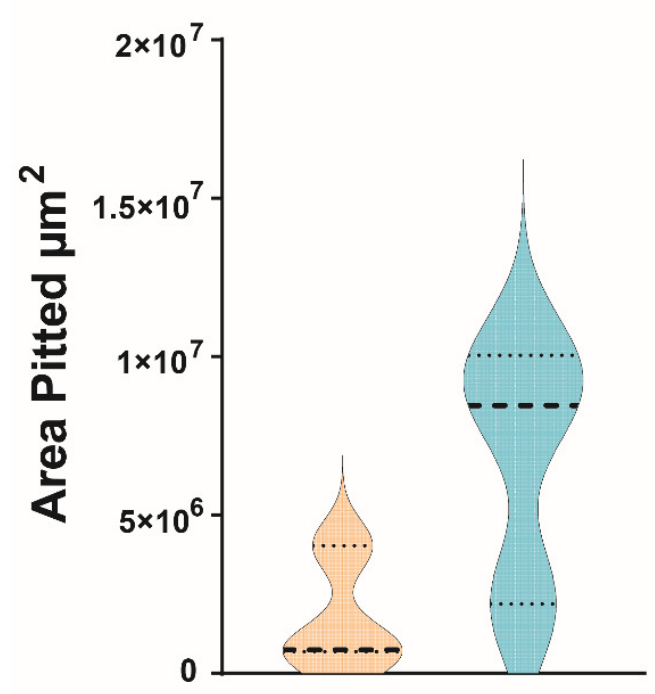

B

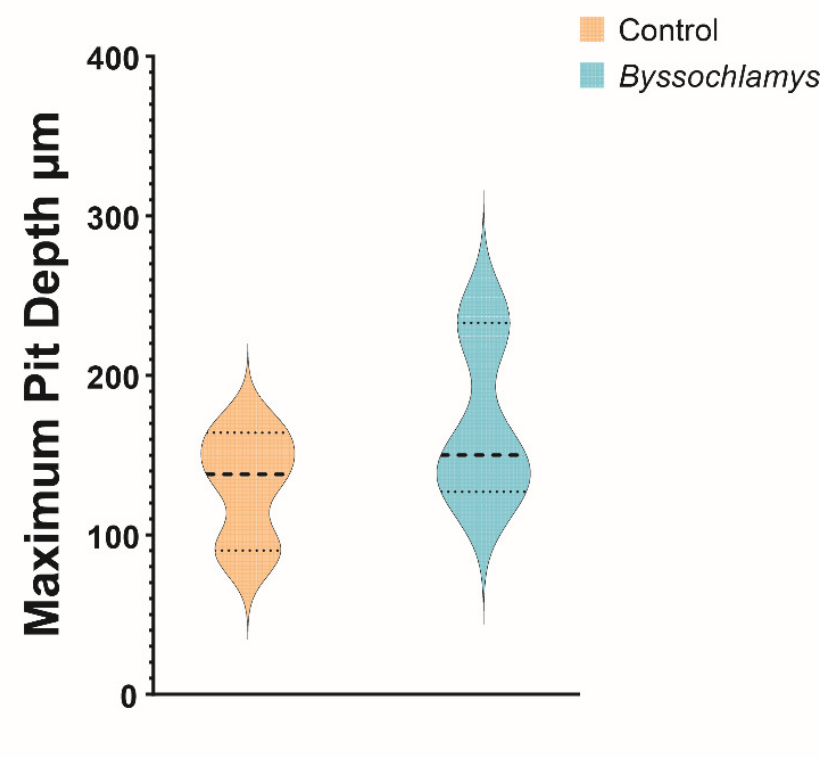

FIG 6. A. Total pitted area on carbon steel coupons (pits $>20 \mu m$ below mean surface average) inoculated with Byssochlamys (Blue) compared to uninoculated controls (Tan). The bold line represents the medium of the data and the nonbold lines represent the data quartiles $(n=3)$. B. Maximum pit depths on carbon steel coupons in abiotic controls (Tan) and flasks inoculated with Byssochlamys (Blue) over 90 days. The bold line represents the median of the data and the nonbold lines represent the data quartiles $(n=3)$. 


\section{Byssochlamys}

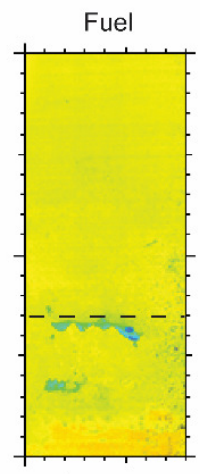

Aqueous

\section{Control}

Fuel

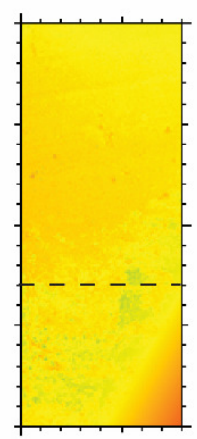

Aqueous

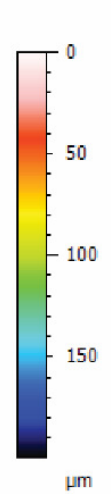

$\mu \mathrm{m}$

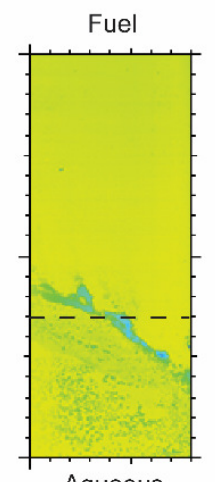

Aqueous

Fuel

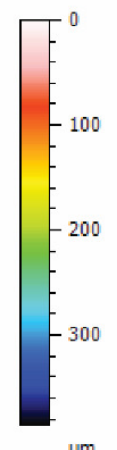

$\mu \mathrm{m}$

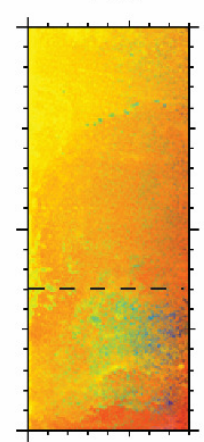

Aqueous

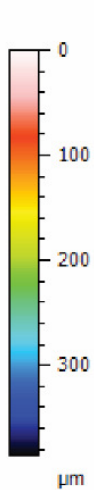

$\mu \mathrm{m}$

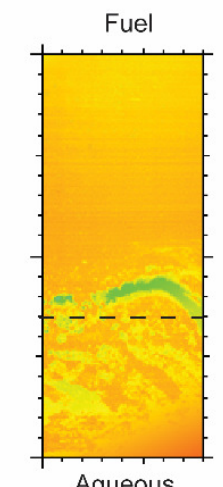

Aqueous

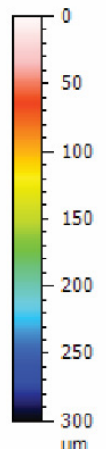

$\mu \mathrm{m}$

Fuel
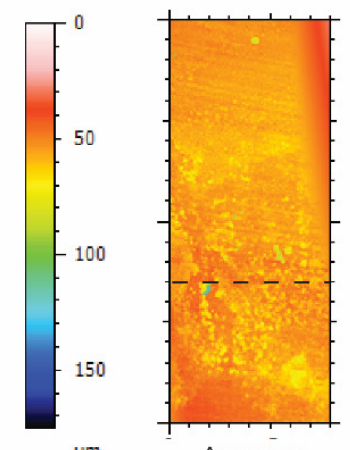

Aqueous

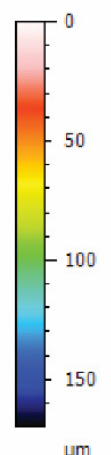

$\mu \mathrm{m}$

FIG 7. Surface depth profiles of carbon steel coupons exposed to the filamentous fungus Byssochlamys and uninoculated controls after 90 days. Biological replicates are show next to each other and depth was calculated from the highest point on the surface of the coupon representing $0 \mu \mathrm{m}$. The color scale bar represents depths in microns. The dashed bar represents where the interface was located on the coupons with the fuel phase above and the aqeous phase below. 


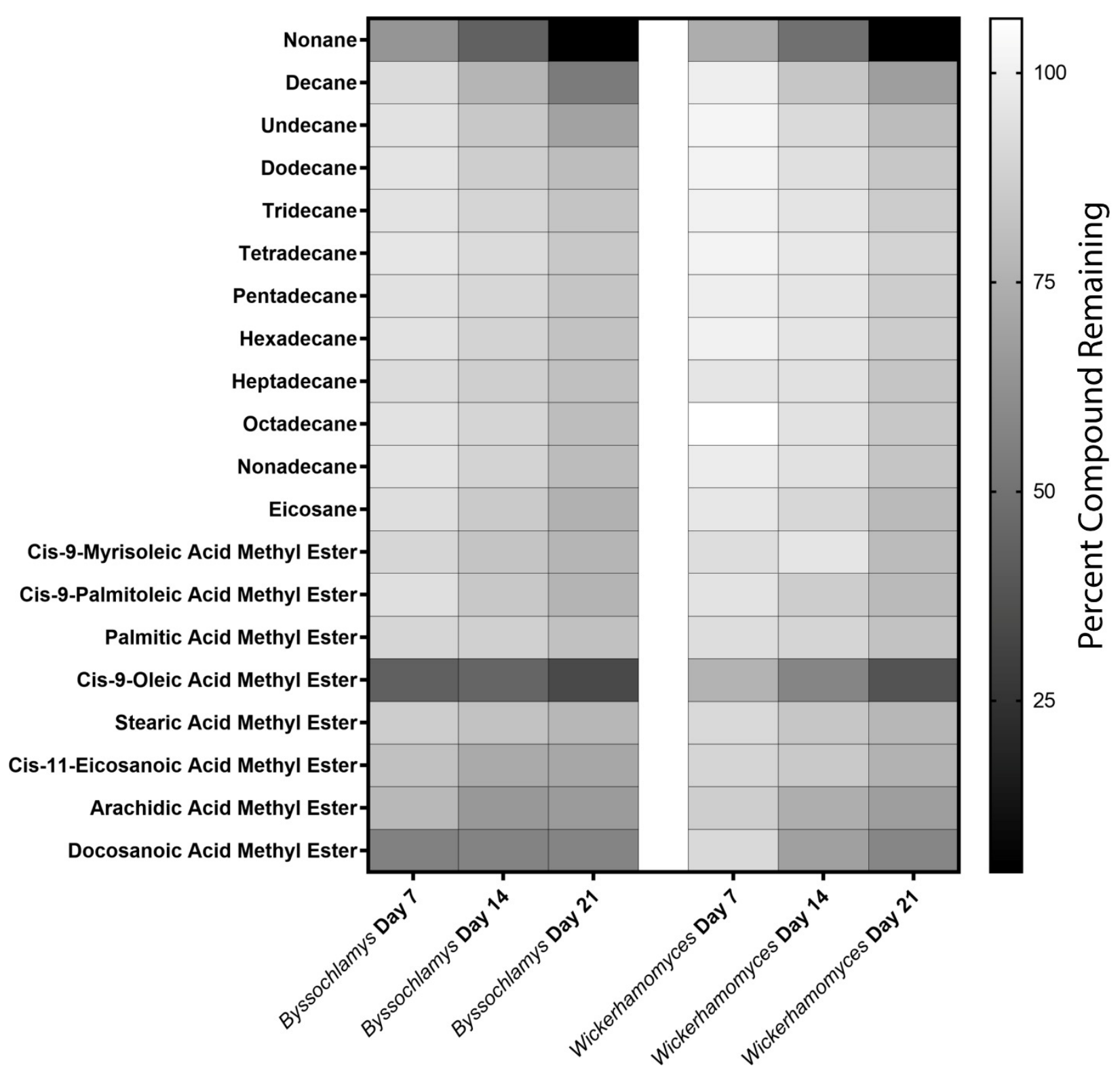

FIG 8. Degradation of alkanes and FAME in B20 biodiesel by Wickerhamomyces and remaining compound compared to the unexposed control with white indicating no degradation and black indicating complete degradation. 
1 3D files used to scan witness coupons at precise locations on using the Nanovea 2 PS $\mathbf{5 0}$

3 https://github.com/Jfloydo/3D-Printed-White-Light-Profilometer-Stage-Cover-for-

$4 \quad$ Nanovea-PS50/tree/main

5 WhiteLightStage_CorrectedBase_BioreactorCouponsForCircularcouponswithDia 6 mter0.5inches.glb

7 Supplementary 3D file for printing a cover over the Nanovea PS50 that supports the 8 placement of circular surface coupons with diameters of $12.7 \mathrm{~mm} / 0.5 \mathrm{in}$.

9 WhiteLightStage_CorrectedBase_3inx0.5in.glb

10 Supplementary 3D file for printing a cover over the Nanovea PS50 that supports the 11 placement of rectangular surface coupons with lengths of $76.2 \mathrm{~mm} / 3 \mathrm{in}$ and widths of $12 \quad 12.7 \mathrm{~mm} / 0.5 \mathrm{in}$. 